\title{
Rhizosphere microorganisms can influence the timing of plant flowering
}

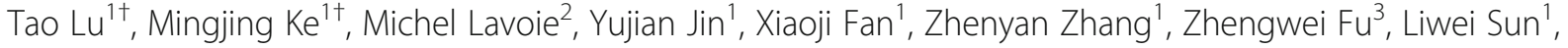
Michael Gillings ${ }^{4}$, Josep Peñuelas ${ }^{5,6}$, Haifeng Qian ${ }^{1,7^{*}}$ (i) and Yong-Guan Zhu ${ }^{8,9^{*}}$

\begin{abstract}
Background: Plant phenology has crucial biological, physical, and chemical effects on the biosphere. Phenological drivers have largely been studied, but the role of plant microbiota, particularly rhizosphere microbiota, has not been considered.

Results: We discovered that rhizosphere microbial communities could modulate the timing of flowering of Arabidopsis thaliana. Rhizosphere microorganisms that increased and prolonged $\mathrm{N}$ bioavailability by nitrification delayed flowering by converting tryptophan to the phytohormone indole acetic acid (IAA), thus downregulating genes that trigger flowering, and stimulating further plant growth. The addition of IAA to hydroponic cultures confirmed this metabolic network.

Conclusions: We document a novel metabolic network in which soil microbiota influenced plant flowering time, thus shedding light on the key role of soil microbiota on plant functioning. This opens up multiple opportunities for application, from helping to mitigate some of the effects of climate change and environmental stress on plants (e.g. abnormal temperature variation, drought, salinity) to manipulating plant characteristics using microbial inocula to increase crop potential.
\end{abstract}

Keywords: Rhizosphere, Microbiota, Root exudate, Nitrogen, Indole acetic acid, Arabidopsis, Flowering time

\section{Background}

Climate change has altered plant phenology $[1,2]$. This has crucial biological, physical, and chemical effects on the biosphere and the earth system [3]. These phenological alterations have become a subject of great interest in ecological and environmental sciences. Changes to phenology have been attributed to multiple factors, including warming [4], but the role of plant microbiota and particularly rhizosphere microbiota has not been considered. And yet, the rhizosphere harbors a diverse community of microorganisms that play critical roles in plant growth and reproduction $[5,6]$.

We know that rhizosphere microbiota protect against pathogens, improve growth by producing phytohormones,

\footnotetext{
* Correspondence: hfqian@zjut.edu.cn; ygzhu@iue.ac.cn

${ }^{\dagger}$ Tao Lu and Mingjing Ke contributed equally to this work.

${ }^{1}$ College of Environment, Zhejiang University of Technology, Hangzhou

310032, People's Republic of China

${ }^{8}$ Key Lab of Urban Environment and Health, Institute of Urban Environment,

Chinese Academy of Sciences, Xiamen 361021, People's Republic of China

Full list of author information is available at the end of the article
}

and may help plants withstand environmental perturbations such as abnormal variation in temperature, drought, and salinity related to climate [7-10]. Recent studies also suggest that root microbiota can contribute to phenotypic plasticity, which has important implications for our understanding of plant phenology in a changing climate and for increasing crop production $[11,12]$. Several auxins have decisive functions in the establishment of plant developmental and reproductive programs $[13,14]$. Auxins can be synthesized by rhizosphere microorganisms $[15,16]$, raising the intriguing possibility that root microbiota may regulate plant growth and development through phytohormone production.

Understanding the interactions between plant microbiota, root exudation, and plant growth and reproduction, however, remains limited, despite significant advances in the last decade [17]. Root exudates account for 5-21\% of total photosynthetically fixed carbon and help drive the composition of rhizosphere communities $[18,19]$. Exudates may be excess plant products $[20,21]$, but they can also contain signaling and chemoattractant molecules. These molecules recruit beneficial microorganisms that 
contribute to pathogen resistance, water retention, and the synthesis of growth-promoting hormones [22], and may influence plant phenotype [23].

Interactions between exudates, soil microbiota, and plant physiology have the potential to dynamically affect rhizospheric communities and alter plant phenotypes by complex feedback mechanisms. We studied the molecular interactions among root exudates, rhizosphere microbiota, and plant physiology in wild-type (Wt) and mutant (pgr5) plants of Arabidopsis thaliana (hereafter Arabidopsis) and identified a novel network of molecular interactions linking the nitrogen cycle, the phytohormone IAA produced from Tryptophan (Trp), and the timing of flowering. These results thus provide evidence of an outstanding phenomenon: the timing of plant flowering may be influenced by soil microbiota.

\section{Results and discussion}

Rhizosphere microbiota can delay the onset of flowering of Wt Arabidopsis

Multiple generations of experimental adaptation/acclimation could be used to observe microbially mediated mechanisms of plant growth and reproduction [24-26]. To test whether the multi-generations of rhizosphere microbiota can induce earlier or delay flowering time, we measured the phenotypic parameters of Arabidopsis plants growing in soil for three generations (G1, G2, or G3) inoculated with different soil microbiomes (Fig. 1a). The phenotypic parameters of Wt Arabidopsis did not change significantly in plants treated with microbiota isolated from the roots of wild-type plants (Wt-M treatment) compared to the corresponding control (growing in sterilized soil without addition of soil microbiomes) during the first two generations (Additional file 1: Table S1). This indicates that plants in sterilized soil could grow as well as those grown in sterilized soil inoculated with a live microbial slurry during the first two generations. Wt-M rhizosphere microbiota significantly affected flowering and reproduction by the third generation. Flowering time in the G3-Wt-M group was significantly delayed, by approximately 3 days, and silique number increased significantly.

The richness and diversity of the rhizosphere microorganisms tended to decrease in parallel with the changes

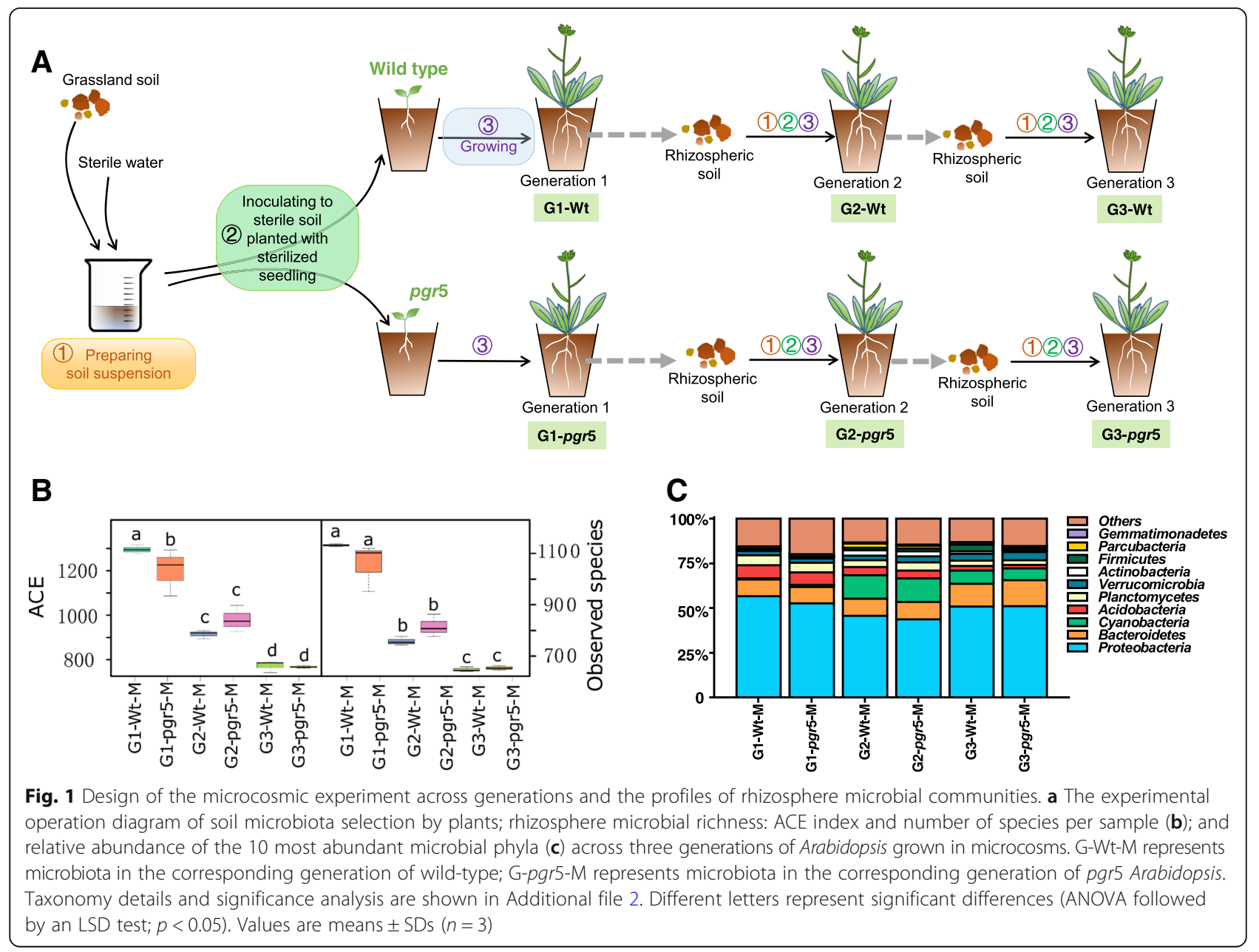


in Arabidopsis physiology over the three generations. Species richness and diversity based on four indices (Chao1, abundance-based coverage (ACE), number of species, and the Shannon index) significantly decreased after the two and three generations (Fig. $1 \mathrm{~b}$ and Additional file 1: Figure S1). The relative abundances of Proteobacteria and Acidobacteria, the dominant bacterial phyla in the rhizosphere, decreased by generations 2 and 3 relative to generation 1. In contrast, Bacteroidetes and cyanobacterial abundances increased by generation 3 (Fig. 1c and Additional file 2). It was demonstrated that some early (or late)-flowering plant could be associated to specific soil microorganism families [25] and that plant microbiota could be associated with changes in different plant growth phases [27]. The results suggest that the selected enriched microbes during the three generations play crucial roles in modulating plant flowering time.

\section{Rhizosphere microbiota advanced the flowering time of the pgr5 mutant}

To better understand the relationships between microbiota, exudates, and flowering time, we used an Arabidopsis mutant (for the PGR5 gene that encodes a novel thylakoid membrane protein) that grows as well as Wt plants in the vegetative phase $[28,29]$. The pgr5 mutant is deficient in antimycin A-sensitive cyclic flow from ferredoxin to plastoquinone, which is one of the most crucial physiological processes for efficient photosynthesis [28]. Because of defects in photosynthesis, the pgr5 mutant produces different exudates from the $\mathrm{Wt}$ Arabidopsis. The phenotype of the pgr 5 mutant was unchanged in generations 1 and 2 in the group treated with pgr 5 microbiota ( $p g r 5-\mathrm{M}$ ) compared to the corresponding control (without the addition of soil microbiomes), as for the Wt Arabidopsis. The flowering time of the pgr5-M-treated group, however, was nearly 4 days earlier by generation 3 , in contrast to $\mathrm{Wt}$, and the silique number was significantly lower (Additional file 1 : Table S1). The changes in plant reproduction induced by the microbial manipulations were probably not driven by rhizosphere diversity, because the Shannon and richness indices of the pgr5-M- and Wt-M-treated groups did not generally differ over the three generations (Additional file 1: Figure S1). The ACE data, though, differed after one generation (Fig. 1b). The relative abundances of the phyla differed only marginally between the two Arabidopsis lines in each generation (Fig. 1c).

\section{Rare rhizosphere microbes potentially affected flowering time}

The abundances of microbial phyla were relatively constant (see above), but abundances between the WM(microbiota from the third-generation Wt cultures) and the PM (microbiota from the third-generation pgr5 cultures)-treated groups differed more at lower taxonomic levels. The relative abundances of 77 rhizosphere genera differed significantly between the WM and PM treatment by generation 3, by at least a factor of two. A total of 41 genera were enriched in the WM treatment relative to the PM treatment, and 36 genera were enriched in the PM treatment (Additional file 1: Table S2 and Additional file 3).

Most of the enriched rhizosphere microorganisms were initially rare (relative abundance $<1 \%$ ), such as Emticicia, Methylobacterium, and Filimonas, suggesting that rare rhizosphere microbes might play a role in modulating Arabidopsis flowering. Rare microbes can be involved in soil biochemical processes and as active modulators of plant growth and resistance to pathogens [30]. The enriched microbes in the WM treatment mostly have key roles in rhizosphere $\mathrm{N}$ regeneration or in maintaining plant growth (Additional file 1: Table S3) [16, 31-34]. Indeed, Bacillus, enriched in the WM treatment, can potentially contribute to soil N fixation [35]. Potential denitrifying organisms such as Stenotrophomonas and Emticicia, though, were enriched in the PM treatment [31, 36]. We hypothesize that an increase in $\mathrm{N}$ fixation and cycling in Wt Arabidopsis associated with the microbiota, and the potential increase in denitrification in the pgr5 mutant, could help increase the duration of N bioavailability in Wt Arabidopsis relative to the pgr5 mutant. Plant pathogenic genera enriched in PM, such as Panacagrimonas and Filimonas, may also contribute to the earlier flowering time, because infected hosts preferentially allocate resources toward reproduction [37, 38]. This hypothesis linking $\mathrm{N}$ availability to flowering time is discussed further below within the newly proposed molecular network that modulates flowering time.

\section{Verification of microbial function}

We unambiguously demonstrated that flowering time was directly associated with the rhizosphere microbiota. Microbiota from the third-generation pgr5 (PM) or Wt cultures (WM) was used to inoculate cultures of three Arabidopsis lines (Wt and two mutants of the photosynthetic apparatus, pgr5 and pnsB4, deficient in cyclic electron flow from NADPH to plastoquinone) for one generation. The microbiota of the third-generation $\mathrm{Wt}$ culture delayed flowering time and increased shoot growth in all cases compared to the treatment using microbiota from the pgr5 cultures. The addition of Wt microbiota delayed flowering by $3.3,5.5$, and 5.7 days in $\mathrm{Wt}$, the pnsB4 mutant, and the pgr5 mutant, respectively (Fig. 2a). The shoot fresh weight of the plants treated with Wt microbiota also increased significantly in the 

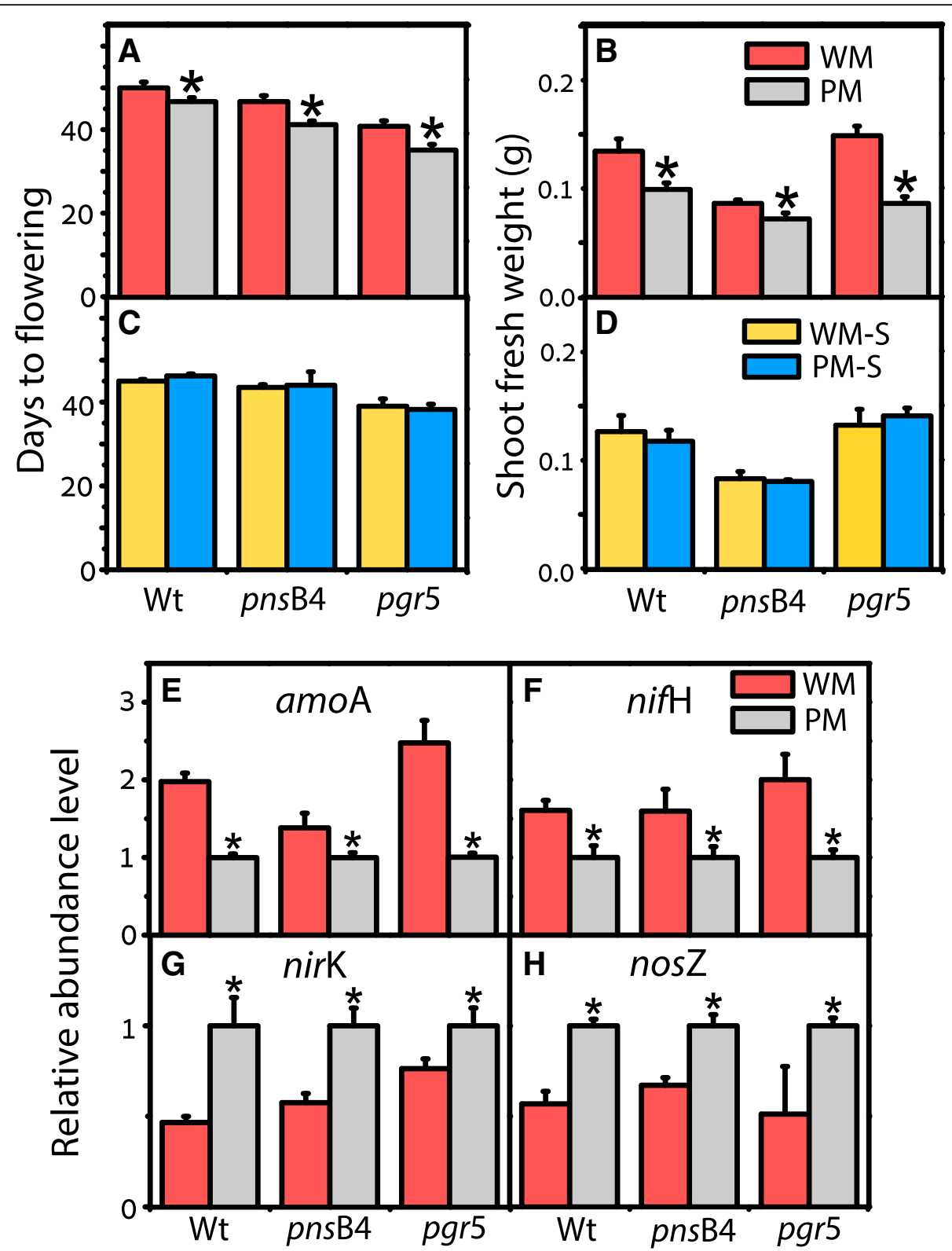

Fig. 2 Verification of microbial function from the third generation. Number of days to the onset of flowering (i.e., when $80 \%$ of the control plants had floral buds of $1 \mathrm{~cm}$ or larger) $(\mathbf{a}, \mathbf{c})$ and shoot fresh weight $(\mathbf{b}, \mathbf{d})$ in wild-type $(\mathrm{Wt})$ and two mutants (pnsB4 and pgr5) of Arabidopsis grown in microcosms in the presence of unsterilized soil slurry (WM and PM) (a, b) or sterilized soil slurry (WM-S and PM-S) (c, d) from the third generation. WM represents plants with microbiota isolates from wild-type plants in generation 3, PM represents the plants with microbiota isolates from pgr5 plants in generation 3, WM-S represents wild-type Arabidopsis grown with sterilized soil slurry, and PM-S represents Arabidopsis pgr5 mutants grown with sterilized soil slurry. Relative abundance of genes involved in N cycling (e amoA, ammonia oxidation; $\mathbf{f}$ nifH, $\mathrm{N}$ fixation; $\mathbf{g}$ nirk, nitrite reductase; $\mathbf{h}$ nosZ, nitrous oxide reductase) in soil samples of Wt and two mutant (pnsB4 and pgr5) lines of Arabidopsis grown in microcosms with the addition of unsterilized rhizosphere soil slurries of wild-type (WM) and mutant (PM) Arabidopsis cultures. Asterisk represents a significant difference between WM and PM $(p<0.05)$. Values are means \pm SDs $(n=9$ with 20 Arabidopsis plants per sample)

three lines compared to the treatments with the addition of pgr5 microbiota (Fig. 2b).

These results clearly indicate that flowering time can be affected by the rhizosphere microbiota. This effect disappeared when the soil slurry was sterilized before inoculation, indicating that heat-stable exudates alone were not modulating Arabidopsis flowering time (Fig. 2c, d). It is interesting that the difference between WMand WM-S (sterilized soil slurry)-treated plants was not very obvious (Additional file 1: Figure S4). The reason may be that sterilized soil slurry included more $\mathrm{N}$, and other nutrients to influence flowering time, which was 
in accordance with the results in Fig. 3. Therefore, we speculated that the metabolites in the sterilized slurry also played a role in influencing the flowering time and plant stature.

The addition of the two soil slurries did not change bulk-soil $\mathrm{pH}$, available soil $\mathrm{K}$ or $\mathrm{P}$ contents (Additional file 1: Table S4), the abundance of key genes involved in the $\mathrm{C}$ cycle, or the activities of $\beta$-glucosidase or chitinase in the rhizosphere (Additional file 1: Figure S2 and S3) but did affect the abundance of genes (normalized to the abundance of 16S rRNA gene) involved in $\mathrm{N}$ cycling (Fig. 2e-h) and the amounts of $\mathrm{NH}_{4}{ }^{+}$ (Fig. 3A) and $\mathrm{NO}_{3}{ }^{-}$(Fig. 3C) in soil. The concentration of bioavailable $\mathrm{N}$ species was generally lower after the addition of the pgr 5 microbiota compared to the treatment with the addition of Wt microbiota (Fig. 3A, C). This decrease in $\mathrm{N}$ availability in the cultures treated with pgr5 microbiota was accompanied by an increase in the abundance of genes involved in denitrification (nirK and nos Z) and a decrease in the abundance of genes involved in nitrogen fixation (nifH) and nitrification (amoA) compared to the Wt-treated groups (Fig. 2e-h). In the Wt-treated group, the activity of urease was higher
(Fig. 3B) and nitrate reductase was lower (Fig. 3D) than those in the PM group, which probably resulted in higher $\mathrm{NH}_{4}{ }^{+}$and $\mathrm{NO}_{3}{ }^{-}$.

Several lines of evidence support the hypothesis that the rhizosphere microbiota modulated $\mathrm{N}$ cycling and bioavailability, leading to $\mathrm{N}$ deficiency earlier in the pgr5-treated groups and thus earlier flowering. Flowering can be triggered by low nitrate levels [39], but plants maximize growth before flowering under the conditions of $\mathrm{N}$ sufficiency [25].

\section{Different root exudates in the two Arabidopsis lines}

Root exudates can act as key substrates or signaling molecules that affect microbial composition [27], so we tested the hypothesis that exudate concentrations and compositions differed between the Arabidopsis lines. A metabolomic analysis found that 34 exudates involved in 10 metabolic pathways were differentially released in the two lines (Wt and pgr5) (see Additional file 1: Table S5, the principal component analysis in Fig. 4a, and $\geq$ 2 - or $\leq 0.5$-fold changes and $p$ values $<0.05$ in Fig. 4b). Four of the 10 biochemical pathways were upregulated in the Wt cultures relative to the pgr5 cultures (Fig. $4 \mathrm{~b}$ ).

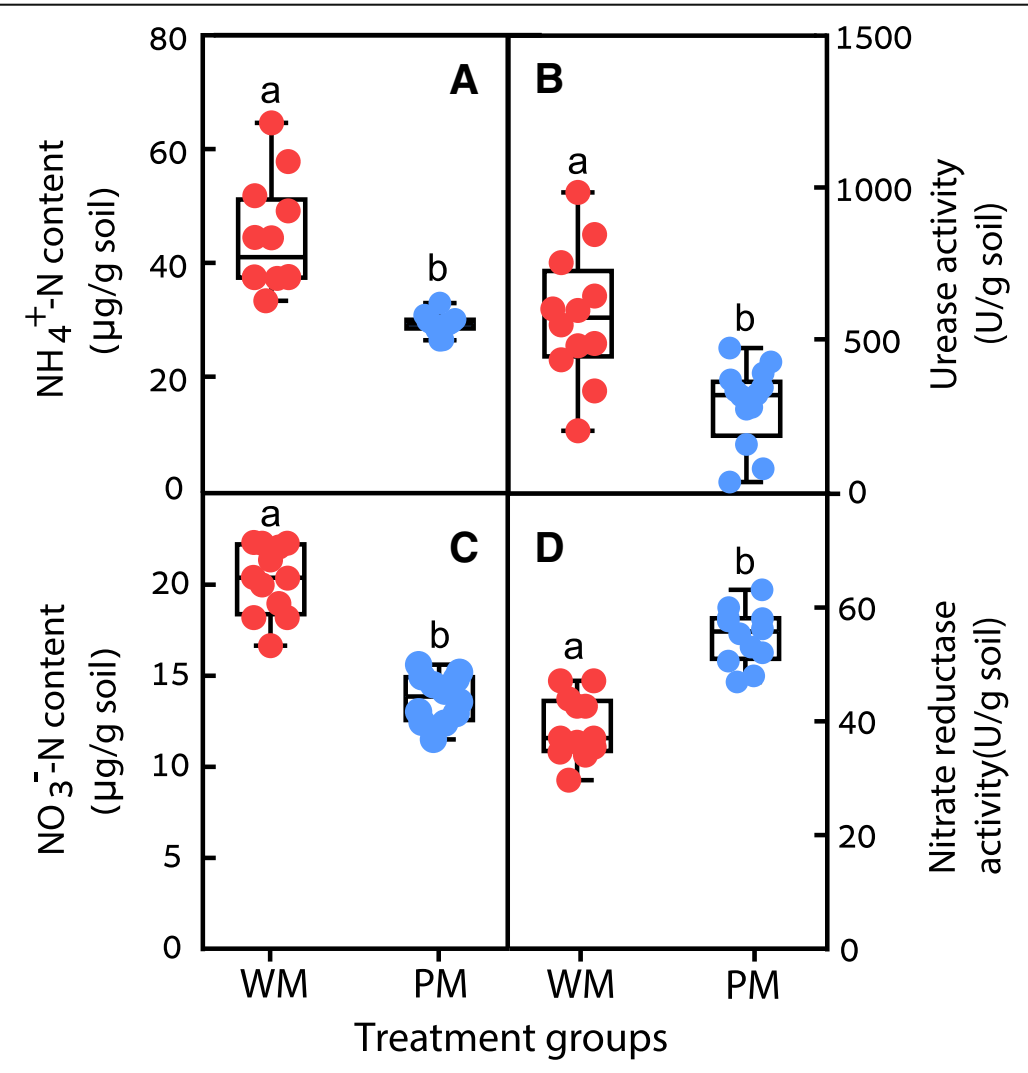

Fig. $3 \mathrm{~N}$ availability could be influenced by the addition of microbiota from the third generation. $\mathrm{NH}_{4}{ }^{+}-\mathrm{N}$ content $(\mathbf{A})$, urease activity $(\mathbf{B}), \mathrm{NO}_{3}{ }^{-}-\mathrm{N}$ content (C), and nitrate reductase activity (D) after inoculation for the WM- and PM-treated groups in the three Arabidopsis lines (Wt, pnsB4, and pgr5) $(n=12)$. Different letters represent significant differences $(p<0.05)$. The red symbols represent the WM-treated samples, and the blue symbols represent the PM-treated samples 

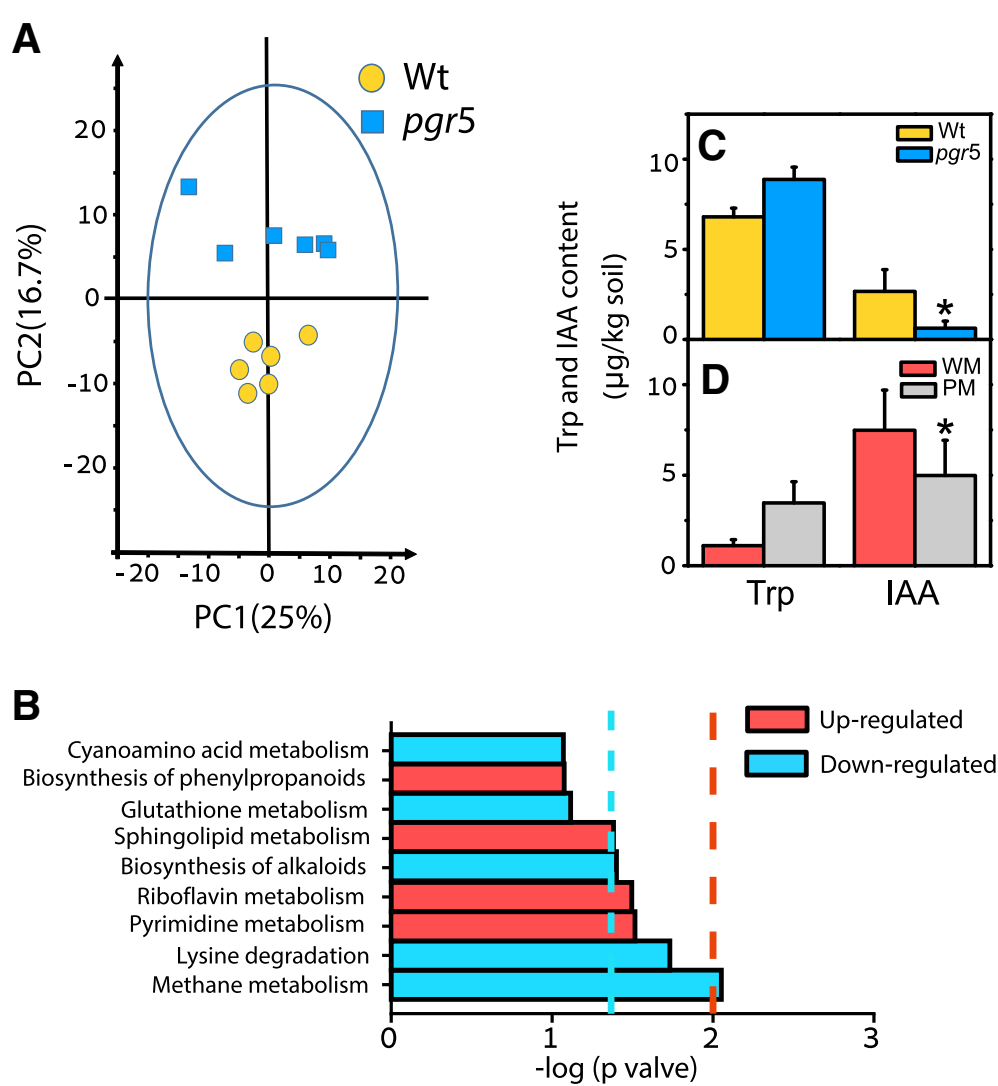

Fig. 4 Root exudates of the two Arabidopsis lines. a Principal component analysis (PCA) of root exudates in the Wt and pgr5 treatments $(n=6)$ grown hydroponically. $\mathbf{b}$ Differentially released exudates $(p<0.05)$ in the Wt and pgr5 treatments were classified into corresponding metabolic pathways inferred from the KEGG pathway database $(n=6)$. c Tryptophan (Trp) and indole-3-acetic acid (IAA) contents in the soil of the cultures of Wt Arabidopsis or pgr5 mutants grown for three generations in microcosms. $\mathbf{d}$ Trp and IAA contents in the soil-cultured Wt Arabidopsis for one generation that had been in advance added the microbiota from the Wt (WM) or the pgr5 mutant (PM) Arabidopsis. Red and blue bars indicate that the exudates for a given pathway are mainly up- or downregulated, respectively, in the Wt cultures relative to the pgr5 mutant cultures. The blue and red vertical dashed lines depict $p=0.05(-\log (0.05)=1.3)$ and $p=0.01(-\log (0.01)=2)$, respectively. Asterisk represents a significant difference $(p<0.05)$. Values are means $\pm \operatorname{SDs}(n=6)$

Thymine was the most differentially released exudate (Additional file 1: Table S5). Thymine can be degraded by bacteria, perhaps accounting for the increase in $\mathrm{NH}_{4}{ }^{+}$content in the WM groups (Fig. 3A).

Trp and its derivatives, phenols, and some carboxylic acids were preferentially exuded in the Wt cultures (Additional file 1: Table S5). The concentrations of amino acids were generally higher in the Wt cultures, consistent with a higher abundance of Bacillus subtilis in the Wt cultures, for which amino acids are chemoattractants. These metabolomic results are consistent with the hypothesis that differences in exudates affect rhizosphere microbiota [40].

\section{IAA delayed flowering time by downregulating genes involved in flowering}

Auxins regulate plant growth in many ways [13, 41, 42]. One of the important auxins for plants is IAA, which is soluble in aqueous solutions and, when protonated, diffuses passively across cell membranes without the need of a specific transporter [43]. IAA has also been hypothesized to have a floral-inductive signaling role by regulating multiple aspects of embryonic and postembryonic development [44, 45]. Microorganisms can produce IAA from Trp [46-49]. One of the enriched rhizosphere microorganisms, Arthrobacter (Additional file 1: Table S3), has been reported to be beneficial for plant growth by having the ability to produce IAA [16]. Trp and its derivatives were enriched in the Wt exudates, so the generation of IAA by microorganisms may control flowering time by a novel molecular network. Trp content in the soil of generation 3 of the Wt cultures decreased, and the IAA content increased 3.03-fold, suggesting that the Wt microbiota rapidly converted Trp into IAA (Fig. 4c, d).

We explored the possibility that microbially generated IAA delayed flowering time by adding Trp, 5hydroxytryptophan (5-HTP), and IAA to the hydroponic cultures and monitoring the flowering time of each 
line. Adding 5 and $25 \mathrm{nM}$ IAA decreased the bolting proportion of Wt Arabidopsis by $20-30 \%$ (Fig. 5a) when $80 \%$ of the control plants had floral buds, implying that IAA was the direct driver that delayed flowering time. Changes in the expression of genes involved in flowering further supported the role of IAA in regulating flowering time (Fig. 5b). IAA treatment induced changes in the relative transcription rates of genes associated with flowering. The rates for some of the genes comprising the autonomous, GA, and vernalization pathways changed significantly, and these changes were observable at the early (when 10\% of control plants had floral buds), intermediate (when $>50 \%$ of control plants had floral buds), and late (when $>80 \%$ of control plants had floral buds) stages of flowering. The downregulation of gene activity was most consistent in the photoperiod pathway, where multiple genes were downregulated at all flowering stages.

Shimada et al. [14] reported that IAA relieved the inhibitory effects of aspterric acid on pollen growth and thus speculated that IAA accelerated reproductive growth in Arabidopsis. To our knowledge, our microcosm study is the first to demonstrate that IAA is one critical signal that delays flowering in Arabidopsis, although Wagner et al. [50] also found a similar phenomenon, while not proposing any related mechanism.

Combined with earlier reports, the weight of evidence suggests that IAA stimulates the development of floral organs but delays the flowering time of Arabidopsis. Our data strongly suggest that IAA delays flowering and acts as a signal of optimal growth conditions in the absence of $\mathrm{N}$ limitation for growth.

\section{Conclusions}

Identifying the community/function relationships for rhizosphere microorganisms and their interaction with plant physiology is critical for determining the role of the plant microbiome in regulating biogeochemical cycles, plant growth, and phenology (Fig. 6). We identified a novel metabolic network in which exudates affect plant rhizosphere microbiota, which can then modulate flowering time by IAA production and can also affect vegetative growth by influencing $\mathrm{N}$ availability. IAA-promoted plant growth is expected to further stimulate exudation and hence retroactively affect flowering time in a positive feedback mechanism. Our results have important implications for our understanding and modeling of plant phenology and are of great interest for the biotechnology sector seeking to increase crop potential.

\section{Methods}

Seedling culture

Arabidopsis seeds (wild-type Col-0 (Wt) and the pgr5 mutant, deficient in PGR5-dependent cyclic electron flow from ferredoxin to plastoquinone), were surface sterilized to avoid bacterial contamination on solid
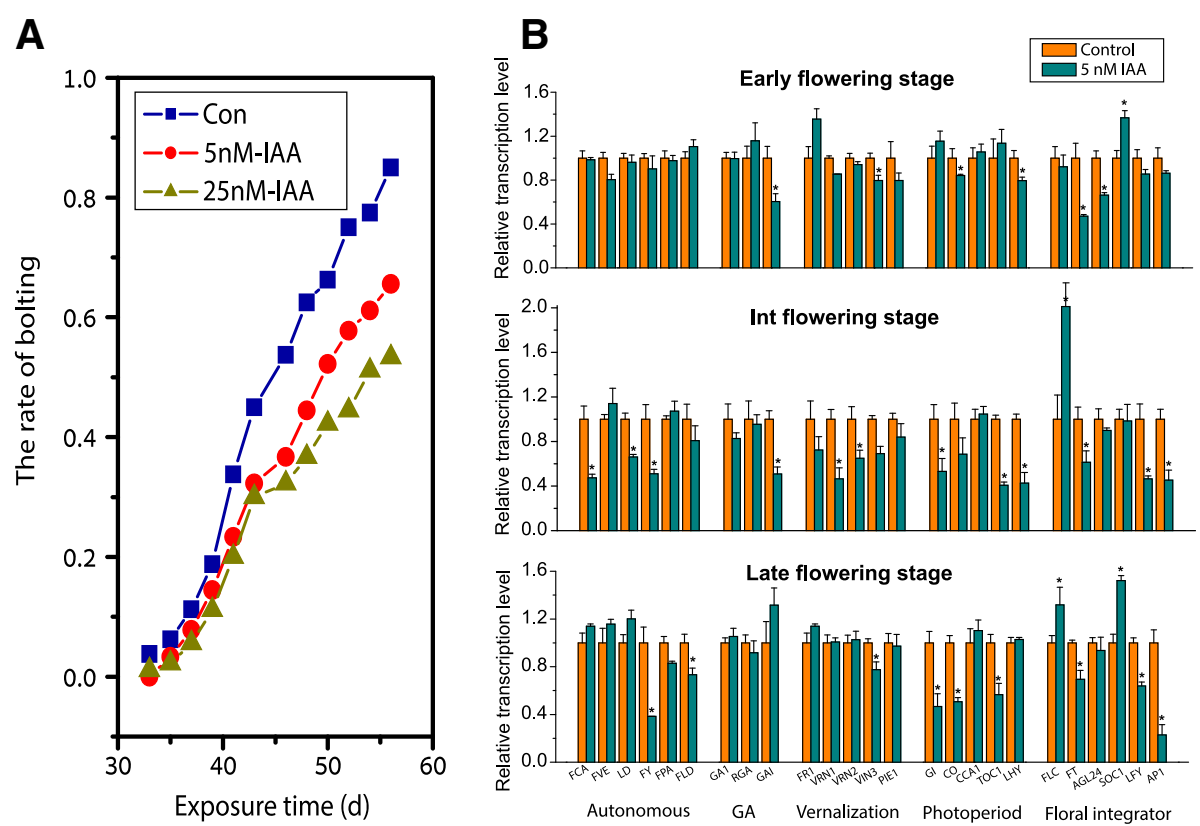

Fig. 5 IAA delayed flowering time by downregulating genes involved in flowering. a Proportion of bolting (i.e., percentage of plants had floral buds of $1 \mathrm{~cm}$ or larger). Plants were hydroponically grown in MS medium containing $5 \mathrm{nM}$ and $25 \mathrm{nM}$ IAA. $\mathbf{b}$ Relative rates of transcription of genes involved in flowering in Wt Arabidopsis at the early, intermediate (Int.), and late stages of flowering after one generation of culture. Plants were hydroponically grown in MS medium containing $5 \mathrm{nM}$ IAA. All transcription levels are normalized to that of a housekeeping gene (actin 2). Values are means \pm SDs $(n=6)$. Asterisk represents a significant difference $(p<0.05)$ 


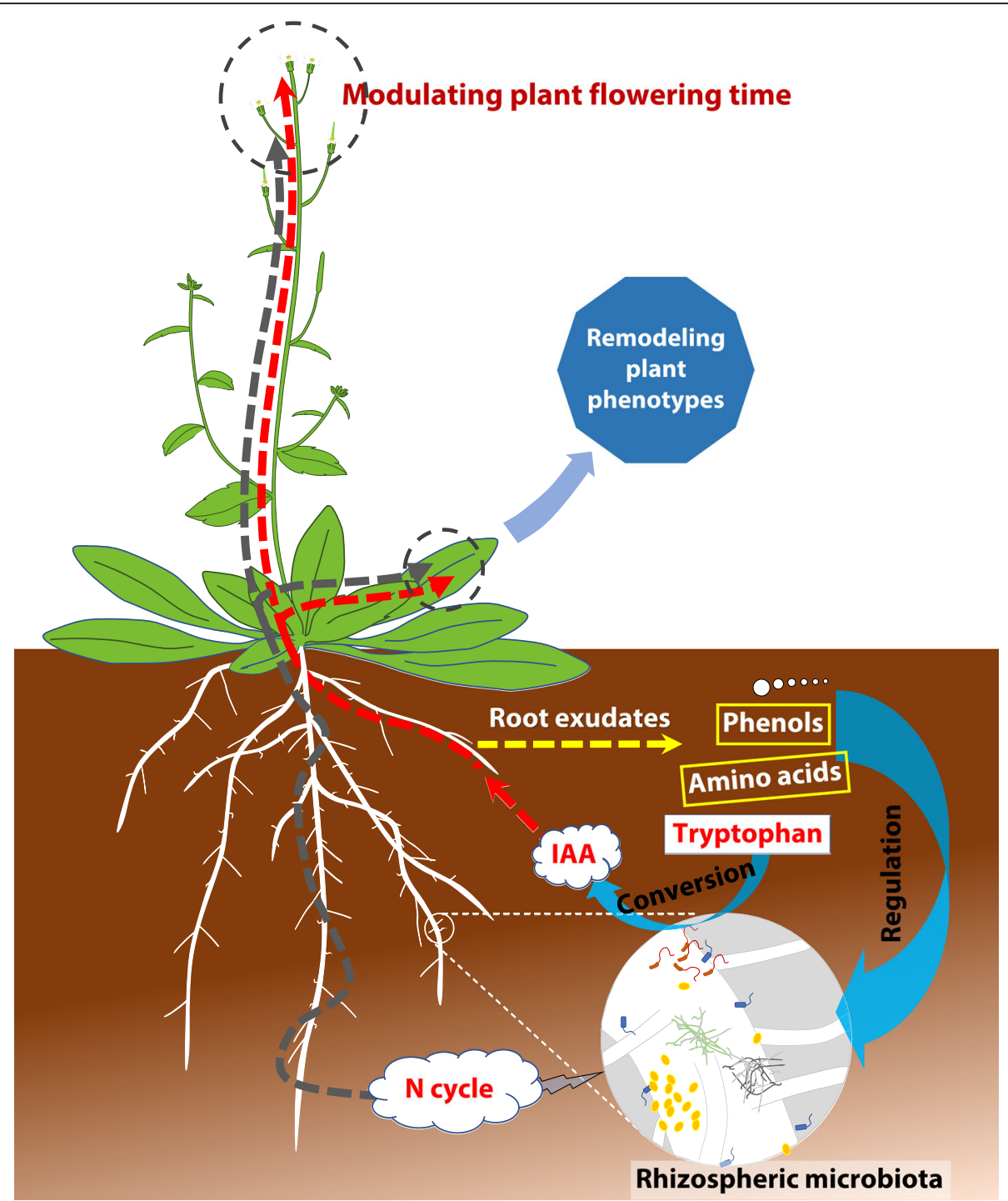

Fig. 6 Schematics of interactions between plants and rhizosphere microbiota. The proposed community/function relationships for rhizosphere microorganisms and their interaction with plant physiology and floral phenology through root exudates, $\mathrm{N}$ cycling, and production of the phytohormone IAA

medium and vernalized as described by Sun et al. [51]. Given that photosynthesis is the main resource of exudates, photosystem mutant with the same genetic background with that of Wt was selected to induce different exudates. Vernalized seeds were cultured in Petri dishes containing Murashige and Skoog (MS) medium under sterile conditions at $25^{\circ} \mathrm{C}$ at a light intensity of $300 \mu \mathrm{mol}$ photons $/ \mathrm{m}^{2} / \mathrm{s}$ and a 12:12-h light to dark photoperiod. The MS medium containing 3\% sugar and 0.5\% agar was autoclaved at $115^{\circ} \mathrm{C}$ for $30 \mathrm{~min}$ before use. Twoweek-old aseptic seedlings were transplanted into polycarbonate pots $(400 \mathrm{~mL})$ containing autoclaved pottingmix soil (Sun Gro Horticulture, MA, USA).

\section{Microcosm experiments across generations}

Approximately $30 \mathrm{~g}$ of grassland soil collected near the Zhejiang University of Technology, China $\left(30^{\circ} 17^{\prime}\right.$ $\left.45.11^{\prime \prime} \mathrm{N}, 120^{\circ} 09^{\prime} 50.07^{\prime \prime} \mathrm{E}\right)$ was mixed with $200 \mathrm{~mL}$ of sterile water by vigorous shaking for $60 \mathrm{~s}$ [25]. Tenmilliliter samples of soil slurry were added to polycarbonate pots transplanted with 20 seedlings of either the Wt or the pgr5 Arabidopsis line. Nine replicate pots were used for generation 1 for each line and for a control treatment $(n=9)$ without added soil slurry. Plants grew in an artificial greenhouse at $25 \pm 0.5^{\circ} \mathrm{C}$ and $80 \%$ relative humidity under cool-white fluorescent light $(300 \mu \mathrm{mol}$ photons $/ \mathrm{m}^{2} / \mathrm{s}$ ) with a 12:12-h light to dark cycle. 
The plants and soils were harvested when $80 \%$ of the plants had floral buds of $1 \pm 0.1 \mathrm{~cm}$ or larger (measured from the center of the rosette), and the time to flowering was recorded. Large soil aggregates that were loosely bound to the roots were first removed by shaking, and $30 \mathrm{~g}$ of the tightly bound rhizosphere soil was then collected (see the "Collection of rhizosphere soil" section) and added to $200 \mathrm{~mL}$ of sterile water, producing a soil slurry for generation 1 . This slurry was used for inoculating the Arabidopsis lines for the second and third generations, as described above. Treatments inoculated with the microbiota of Wt and the pgr5 mutant were designated WM and PM, respectively. The generations in our study refer to the propagation of soil microbes only, and all seeds were from a common stock. At flowering stage and harvest time, the physiological parameters including the number of days before flowering, fresh weight, number of rosette leaves, and number of siliques were determined in wild-type (Wt) and pgr5 mutant ecotypes grown in microcosms for each generation.

\section{Collection of rhizosphere soil}

Rhizosphere soil was collected as described by Bulgarelli et al. [9]. Four-centimeter sections of roots were collected immediately below the rosette, and the roots from each pot were transferred to a 50-mL centrifuge tube containing $20 \mathrm{~mL}$ of sterile phosphate buffer saline (PBS; $137 \mathrm{mM}$ sodium chloride, $10 \mathrm{mM}$ phosphate buffer, 2.7 $\mathrm{mM}$ potassium chloride; $\mathrm{pH} 7.3-7.5)$. The centrifuge tubes were shaken at $40 \mathrm{~g}$ for $20 \mathrm{~min}$ on an orbital shaker. The roots were removed, the solution was centrifuged at $1000 \mathrm{~g}$ for $20 \mathrm{~min}$, and the pellet of rhizosphere soil was recovered. All samples of rhizosphere soil were frozen in liquid nitrogen and stored at $-80^{\circ} \mathrm{C}$ until analysis.

\section{S rRNA gene sequencing}

The frozen soil samples were thawed on ice, and DNA was extracted using a PowerSoil DNA Isolation Kit (MO BIO Laboratories, Inc., Carlsbad, USA). 16S rRNA genes were amplified using EXtaq enzyme (TaKaRa, Kyoto, Japan) and the specific primers 314F (5'-CCTA CGGGNGGCWGCAG-3') and 805R (5'-GACTACHV GGGTATCTAATCC-3') with the adapter (index) that targets the V3 and V4 variable regions of bacterial/archaeal $16 \mathrm{~S}$ rRNA genes. Strongly amplified products 460 bp in length were chosen for further experiments. The amplicons were quantified with a Qubit 2.0 fluorometer (Thermo Fisher Scientific, USA), diluted to $1 \mathrm{ng} / \mu \mathrm{L}$, and sequenced on a MiSeq platform (PE250). In total, 854,514 raw reads were obtained from the 18 samples of rhizosphere soils (6 groups as shown in Fig. 1a, $n=3$ ). We computed operational taxonomic units (OTU) and microbial diversity as described previously [52]. Rarefaction curves of observed species are shown in Additional file 1: Fig. S5.

\section{Analysis of rhizosphere DNA abundance by quantitative real-time PCR (qRT-PCR)}

Rhizosphere DNA was quantified by ultraviolet-visible spectrophotometry (ND 5000, BioTeke, China) and diluted to $10 \mathrm{ng} / \mu \mathrm{L}$. The abundances of rhizosphere genes (amoA, nif $\mathrm{H}$, nirK, nos $\mathrm{Z}, c b b \mathrm{~L}, \beta$-glu, and $C h i \mathrm{~A}$; Additional file 1: Table S6) were measured in 10- $\mu \mathrm{L}$ PCRs containing $5 \mu \mathrm{L}$ of SYBR Green Real-time PCR Master Mix (Toyobo, Tokyo, Japan), $0.4 \mu \mathrm{L}$ of each of the forward and reverse primers $(10 \mathrm{mM})$ (see Additional file 1: Table S6 for a list of primers), $1 \mu \mathrm{L}$ of rhizosphere DNA at 10 $\mathrm{ng} / \mathrm{L}$, and $3.2 \mu \mathrm{L}$ of $\mathrm{ddH}_{2} \mathrm{O}$. The qRT-PCR protocol was $94^{\circ} \mathrm{C}$ for $3 \mathrm{~min}$ followed by 40 cycles of $94{ }^{\circ} \mathrm{C}$ for $30 \mathrm{~s}, 56^{\circ}$ $\mathrm{C}$ for $30 \mathrm{~s}$, and $72{ }^{\circ} \mathrm{C}$ for $1 \mathrm{~min}$ on an Eppendorf MasterCycler ep RealPlex (Wesseling-Berzdorf, Germany).

\section{Measurements in the dissolved phase of the soil samples} Physicochemical (nutrient contents and $\mathrm{pH}$ ) and biological (enzymatic activities and concentrations of IAA and Trp) variables were measured in the dissolved phase of the soil samples. After three generations of Arabidopsis cultures (Fig. 1a), soil samples were then collected randomly from each pot, air-dried, homogenized, and sieved to obtain particles $<1 \mathrm{~mm}$. The activity of soil enzymes ( $\beta$-glucosidase, chitinase, urease, and nitrate reductase) and soil $\mathrm{N}$ content $\left(\mathrm{NH}_{4}{ }^{+}\right.$and $\mathrm{NO}_{3}{ }^{-}$contents) were subsequently measured using $0.5 \mathrm{~g}$ of soil $(n=4)$ following the manufacturer's instructions of corresponding commercial reagent kits (COMIN, Suzhou, China). The concentrations of two key metabolites (IAA and Trp) were measured in the soil dissolved phase. Five grams of soil was mixed with $5 \mathrm{~g}$ of $\mathrm{NaCl}$ and $20 \mathrm{~mL}$ of acetonitrile for $5 \mathrm{~min}$, followed by centrifugation at $1000 \mathrm{~g}$ for $10 \mathrm{~min}$. The concentrations of IAA and Trp in the supernatants were measured by Liquid ChromatographyElectrospray Ionization-Mass/Mass Spectroscopy (LCESI-MS/MS, Q-Trap 5500, Agilent Technologies, USA).

\section{Collection, measurement, and identification of root exudates}

Arabidopsis seedlings (Wt and pgr5 mutant) reached the bolting stage after approximately 40 days of culture and were then transferred to glass containers containing 40 $\mathrm{mL}$ of $\mathrm{ddH}_{2} \mathrm{O}$ [27]. After 3 days of culture in the glass containers, culture media containing exudates were passed through $0.45-\mu \mathrm{m}$ filter membranes. Approximately $35 \mathrm{~mL}$ of the solution was freeze-dried, dissolved in $500 \mu \mathrm{L}$ of $80 \%$ methanol, and derivatized with Bis (trimethylsilyl) trifluoroacetamide and 1\% chlorotrimethylsilane. The derivatized samples were analyzed by gas chromatography/mass spectroscopy GC-MS (Agilent 
7890B gas chromatographic system coupled to an Agilent 5977A Mass Spectrometry Detector (Agilent, USA)). The potential involvement of all identified exudates in Arabidopsis biochemical pathways was subsequently determined by reference to the KEGG database [52].

\section{Addition of Trp, 5-hydroxytryptophan (5-HTP), and IAA and analysis of gene transcription associated with flowering}

For hydroponic experiments (i.e., plants cultured in solution), Trp, 5-HTP, and IAA were added to autoclaved liquid MS medium cultured with Wt at final concentrations of $0.5,5$, and $25 \mathrm{nM}$, respectively. Control cultures without Trp, 5-HTP, and IAA were performed in parallel. The transcription of genes involved in flowering was measured by qRT-PCR. Control groups treated with Trp, 5-HTP, and IAA were analyzed at three stages of development: (1) early flowering (when $10 \%$ of control plants had floral buds), (2) intermediate flowering (when $>50 \%$ of control plants had floral buds), and (3) late flowering (when $>80 \%$ of control plants had floral buds) stages. Total RNA was isolated from the plants and then reverse transcribed into cDNA for qRT-PCR analysis using the protocol described by Chen et al. [11] and the primer pairs in Additional file 1: Table S6. We studied the transcription of six genes in the autonomous pathway (FCA, FLD, FPA, FVE, FY, and LD), three genes in the gibberellin acid (GA) pathway (GAI, GA1, and RGA), six genes in the vernalization pathway (FR1, VRN1, VRN2, VIN3, and PIE1), five genes in the photoperiod pathway (CCA1, CO, GI, LHY, and TOC1), and five genes in the floral integrator pathway (FLC, FT, SOC1, AGL-24, LFY, and AP1).

\section{Data analysis and statistical methods}

For the biochemical and physiological measurements, analysis of variance followed by the Dunnett's post hoc test was performed to evaluate the statistical significance among data using the StatView 5.0 program (Statistical Analysis Systems Institute, Cary, NC, USA). Means among treatments were considered significantly different, when the probability $(p)$ was less than 0.05 . All analyses were performed in triplicate unless otherwise stated. All data are presented in the tables and figures as mean $\pm \mathrm{SD}$ (standard deviation).

\section{Additional files}

Additional file 1: Figure S1. Rhizosphere microbiota richness and diversity. Figure S2. Abundance of carbon cycle-related genes in rhizosphere soil. Figure S3. Activities of carbon cycle-related enzymes in rhizosphere soil. Figure S4. Comparisons between WM- and WMS-treated plants. Figure S5. Rarefaction curves of observed species. Table S1. Physiological parameters of Arabidopsis in three generations. Table S2. Significant enrichment of rhizosphere microorganisms in the third generation. Table S3. nriched rare microorganisms in Wt and pgr5 Arabidopsis. Table S4. Bulk soil properties measured after addition of WM and PM soil slurries. Table S5. Comparison of root exudates between Wt and pgr5 mutant Arabidopsis. Table S6. Sequences of the primer pairs used for qRT-PCR. (DOCX 348 kb)

Additional file 2: Taxonomy (relative abundance) of rhizosphere microorganisms at the phylum level. (XLSX $35 \mathrm{~kb}$ )

Additional file 3: Taxonomy (relative abundance) of rhizosphere microorganisms at the genus level. (XLSX $46 \mathrm{~kb}$ )

\section{Acknowledgements}

We sincerely thank all the authors who provided valuable data or comments for this study. We are very thankful to Dr. Tsuyoshi Endo and Professor Fumihiko Sato (Kyoto University, Japan) who provided the pnsB4 and pgr5 mutant lines of Arabidopsis thaliana.

\section{Funding}

This work was financially supported by the National Natural Science Foundation of China (21577128, 21777144), the Strategic Priority Research Program of the Chinese Academy of Sciences (XDB15020302 and XDB15020402), the CAS Pioneer Hundred Talents Program (H.F. Qian), and the Xingjiang Uighur Autonomous Region Talent Project (H.F. Qian). JP research is funded by the European Research Council Synergy grant SyG-2013-610028 IMBALANCE-P.

\section{Availability of data and materials}

All data needed to evaluate the conclusions in the paper are present in the paper and/or the supplementary materials. The raw data of 165 rRNA gene sequences have been submitted to the NCBI Sequence Read Archive (SRA) database under the BioProject number PRJNA494813.

\section{Authors' contributions}

HFQ, ZWF, and YGZ designed the study; TL, MJK, YJJ, XJF, and ZYZ performed the research; HFQ, TL, MJK, YJJ, LWS, and YGZ analyzed the data; and HFQ, TL, YJJ, ML, MG, JP, and YGZ wrote the manuscript. All authors read and approved the final manuscript.

Ethics approval and consent to participate

Not applicable.

Consent for publication

Not applicable.

\section{Competing interests}

The authors declare that they have no competing interests.

\section{Publisher's Note}

Springer Nature remains neutral with regard to jurisdictional claims in published maps and institutional affiliations.

\section{Author details}

${ }^{1}$ College of Environment, Zhejiang University of Technology, Hangzhou 310032, People's Republic of China. ${ }^{2}$ Quebec-Ocean and Takuvik Joint International Research Unit, Université Laval, Québec G1VOA6, Canada. ${ }^{3}$ College of Biotechnology and Bioengineering, Zhejiang University of Technology, Hangzhou 310032, People's Republic of China. ${ }^{4}$ Department of Biological Sciences, Macquarie University, Sydney, NSW 2109, Australia. ${ }^{5}$ CSIC, Global Ecology Unit, CREAF-CSIC-UAB, Barcelona, Catalonia, Spain. ${ }^{6}$ CREAF, Cerdanyola del Vallès, Barcelona, Catalonia, Spain. ${ }^{7}$ Xinjiang Key Laboratory of Environmental Pollution and Bioremediation, Xinjiang Institute of Ecology and Geography, Chinese Academy of Sciences, Urumqi 830011, People's Republic of China. ${ }^{8}$ Key Lab of Urban Environment and Health, Institute of Urban Environment, Chinese Academy of Sciences, Xiamen 361021, People's Republic of China. ${ }^{9}$ State Key Lab of Urban and Regional Ecology, Research Center for Ecoenvironmental Sciences, Chinese Academy of Sciences, Beijing 100085, People's Republic of China. 
Received: 15 September 2018 Accepted: 17 December 2018 Published online: 26 December 2018

\section{References}

1. Penuelas J, Filella I. Phenology. Responses to a warming world. Science. 2001;294:793-5.

2. Fitter $\mathrm{AH}$, Fitter RSR. Rapid changes in flowering time in British plants. Science. 2002;296:1689-91.

3. Penuelas J, Rutishauser T, Filella I. Phenology feedbacks on climate change. Science. 2009;324:887-8.

4. Menzel A, Sparks TH, Estrella N, Koch E, Aasa A, Ahas R, et al. European phenological response to climate change matches the warming pattern. Glob Chang Biol. 2006;12:1969-76.

5. Hinsinger $P$, Bengough AG, Vetterlein D, Young IM. Rhizosphere: biophysics, biogeochemistry and ecological relevance. Plant Soil. 2009;321:117-52.

6. Haney CH, Samuel BS, Bush J, Ausubel FM. Associations with rhizosphere bacteria can confer an adaptive advantage to plants. Nat Plants. 2015;1:1-9.

7. Lu T, Ke MJ, Peijnenburg WJGM, Zhu YC, Zhang M, Sun LW, et al. Investigation of rhizospheric microbial communities in wheat, barley, and two rice varieties at the seedling stage. J Agr Food Chem. 2018;66:2645-53.

8. Innerebner G, Knief C, Vorholt JA. Protection of Arabidopsis thaliana against leaf-pathogenic Pseudomonas syringae by Sphingomonas strains in a controlled model system. Appl Environ Microb. 2011;77:3202-10.

9. Bulgarelli D, Rott M, Schlaeppi K, van Themaat EVL, Ahmadinejad N, Assenza F, et al. Revealing structure and assembly cues for Arabidopsis root-inhabiting bacterial microbiota. Nature. 2012:488:91-5.

10. Peiffer JA, Spor A, Koren O, Jin Z, Tringe SG, Dangl JL, et al. Diversity and heritability of the maize rhizosphere microbiome under field conditions. P Natl Acad Sci USA. 2013;110:6548-53.

11. Chen S, Li XX, Lavoie M, Jin YJ, Xu JH, Fu ZW, et al. Diclofop-methyl affects microbial rhizosphere community and induces systemic acquired resistance in rice. J Environ Sci-China. 2017:51:352-60.

12. Singh BK, Bardgett RD, Smith P, Reay DS. Microorganisms and climate change: terrestrial feedbacks and mitigation options. Nat Rev Microbiol. 2010:8:779-90.

13. Alabadi D, Blazquez MA, Carbonell J, Ferrandiz C, Perez-Amador MA. Instructive roles for hormones in plant development. Int J Dev Biol. 2009;53:1597-608

14. Shimada A, Yamane $H$, Kimura $Y$. Interaction between aspterric acid and indole-3-acetic acid on reproductive growth in Arabidopsis thaliana. Z Naturforsch C. 2005;60:572-6.

15. Treesubsuntorn C, Dhurakit P, Khaksar G, Thiravetyan P. Effect of microorganisms on reducing cadmium uptake and toxicity in rice (Oryza sativa L.). Environ Sci Pollut Res Int. 2018;25:25690-701.

16. Li MS, Guo R, Yu F, Chen X, Zhao HY, Li HX, et al. Indole-3-acetic acid biosynthesis pathways in the plant-beneficial bacterium Arthrobacter pascens ZZ21. Int J Mol Sci. 2018;19:E443.

17. Bai $Y$, Muller DB, Srinivas $G$, Garrido-Oter R, Potthoff $E$, Rott $M$, et al. Functional overlap of the Arabidopsis leaf and root microbiota. Nature. 2015; 528:364-9.

18. Vandenkoornhuyse P, Quaiser A, Duhamel M, Le Van A, Dufresne A. The importance of the microbiome of the plant holobiont. New Phytol. 2015; 206:1196-206

19. Mendes R, Garbeva P, Raaijmakers JM. The rhizosphere microbiome: significance of plant beneficial, plant pathogenic, and human pathogenic microorganisms. FEMS Microbiol Rev. 2013:37:634-63.

20. Preece C, Farre-Armengol G, Llusia J, Penuelas J. Thirsty tree roots exude more carbon. Tree Physiol. 2018;38:690-5.

21. Preece C, Penuelas J. Rhizodeposition under drought and consequences for soil communities and ecosystem resilience. Plant Soil. 2016;409:1-17.

22. Berendsen RL, Pieterse CMJ, Bakker PAHM. The rhizosphere microbiome and plant health. Trends Plant Sci. 2012;17:478-86.

23. Cook RJ, Thomashow LS, Weller DM, Fujimoto D, Mazzola M, Bangera G, et al. Molecular mechanisms of defense by rhizobacteria against root disease. P Natl Acad Sci USA. 1995;92:4197-201.

24. Swenson W, Wilson DS, Elias R. Artificial ecosystem selection. P Natl Acad Sci USA. 2000:97:9110-4

25. Panke-Buisse K, Poole AC, Goodrich JK, Ley RE, Kao-Kniffin J. Selection on soil microbiomes reveals reproducible impacts on plant function. ISME 2015:9:980-9.
26. Yuan J, Zhao J, Wen T, Zhao ML, Li R, Goossens P, et al. Root exudates drive the soil-borne legacy of aboveground pathogen infection. Microbiome. 2018;6:156.

27. Chaparro JM, Badri DV, Vivanco JM. Rhizosphere microbiome assemblage is affected by plant development. ISME J. 2014;8:790-803.

28. Munekaga Y, Hashimoto M, Miyaka C, Tomizawa Kl, Endo T, Tasaka M, et al. Cyclic electron flow around photosystem I is essential for photosynthesis. Nature. 2004:429:579-82

29. Qian HF, Tsuji T, Endo T, Sato F. PGR5 and NDH pathways in photosynthetic cyclic electron transfer respond differently to sublethal treatment with photosystem-interfering herbicides. J Agr Food Chem. 2014;62:4083-9.

30. Jousset A, Bienhold C, Chatzinotas A, Gallien L, Gobet A, Kurm V, et al. Where less may be more: how the rare biosphere pulls ecosystems strings. ISME J. 2017;11:853-62.

31. Xie BZ, Liu BJ, Yi Y, Yang L, Liang DW, Zhu Y, et al. Microbiological mechanism of the improved nitrogen and phosphorus removal by embedding microbial fuel cell in anaerobic-anoxic-oxic wastewater treatment process. Bioresour Technol. 2016;207:109-17.

32. Cheng JZ, Li YL, Gao WC, Chen Y, Pan WJ, Lee XQ, et al. Effects of biochar on cd and $\mathrm{Pb}$ mobility and microbial community composition in a calcareous soil planted with tobacco. Biol Fert Soils. 2018:54:373-83.

33. Sy A, Giraud E, Jourand P, Garcia N, Willems A, de Lajudie P, et al. Methylotrophic Methylobacterium bacteria nodulate and fix nitrogen in symbiosis with legumes. J Bacteriol. 2001;183:214-20.

34. Zakhia F, Jeder H, Willems A, Gillis M, Dreyfus B, de Lajudie P. Diverse bacteria associated with root nodules of spontaneous legumes in Tunisia and first report for nifH-like gene within the genera Microbacterium and Starkeya. Microbial Ecol. 2006:51:375-93.

35. Upadhyay SK, Singh DP, Saikia R. Genetic diversity of plant growth promoting rhizobacteria ssolated from rhizospheric soil of wheat under saline condition. Curr Microbiol. 2009;59:489-96.

36. Costa C, Dijkema C, Friedrich M, Garcia-Encina P, Fernandez-Polanco F, Stams AJM. Denitrification with methane as electron donor in oxygenlimited bioreactors. Appl Microbiol Biot. 2000;53:754-62.

37. Korves TM, Bergelson J. A developmental response to pathogen infection in Arabidopsis. Plant Physiol. 2003;133:339-47.

38. Agnew P, Koella JC, Michalakis Y. Host life history responses to parasitism. Microbes Infect. 2000;2:891-6.

39. Ishioka N, Tanimoto S, Harada H. Roles of nitrogen and carbohydrate in floralbud formation in Pharbitis apex cultures. J Plant Physiol. 1991;138:573-6.

40. Hu LF, Robert CAM, Cadot S, Zhang X, Ye M, Li BB, et al. Root exudate metabolites drive plant-soil feedbacks on growth and defense by shaping the rhizosphere microbiota. Nat Commun. 2018;9:2E738.

41. Kunkel BN, Harper CP. The roles of auxin during interactions between bacterial plant pathogens and their hosts. J Exp Bot. 2018;69:245-54.

42. Spaepen S, Bossuyt S, Engelen K, Marchal K, Vanderleyden J. Phenotypical and molecular responses of Arabidopsis thaliana roots as a result of inoculation with the auxin-producing bacterium Azospirillum brasilense. New Phytol. 2014; 201:850-61.

43. Spaepen S, Vanderleyden J, Remans R. Indole-3-acetic acid in microbial and microorganism-plant signaling. FEMS Microbiol Rev. 2007;31:425-48.

44. Spaepen S, Vanderleyden J. Auxin and plant-microbe interactions. CSH Perspect Biol. 2011:3:a001438.

45. Prigge MJ, Greenham K, Zhang Y, Santner A, Castillejo C, Mutka A, et al. The Arabidopsis auxin receptor F-box proteins AFB4 and AFB5 are required for response to the synthetic auxin picloram. G3-Genes Genom Genet. 2016:6:1383-90.

46. Patten $\mathrm{CL}$, Blakney AJC, Coulson TJD. Activity, distribution and function of indole-3-acetic acid biosynthetic pathways in bacteria. Crit Rev Microbiol. 2013;39:395-415.

47. Molina R, Rivera D, Mora V, Lopez G, Rosas S, Spaepen S, et al. Regulation of IAA biosynthesis in Azospirillum brasilense under environmental stress conditions. Curr Microbiol. 2018;75:1408-18.

48. Duca D, Lorv J, Patten C, Rose D, Glick BR. Indole-3-acetic acid in plantmicrobe interactions. Anton Leeuw Int J G. 2014;106:85-125.

49. Patten $\mathrm{CL}$, Glick BR. Bacterial biosynthesis on indole-3-acetic acid. Can J Microbil. 1996;42:207-20

50. Wagner MR, Lundberg DS, Coleman-Derr D, Tringe SG, Dangl JL, MitchellOlds T. Natural soil microbes alter flowering phenology and the intensity of selection on flowering time in a wild Arabidopsis relative. Ecol Lett. 2014;17:717-26. 
51. Sun $C C$, Chen $S$, Jin YJ, Song H, Ruan SL, Fu ZW, et al. Effects of the herbicide imazethapyr on photosynthesis in PGR5-and NDH-deficient Arabidopsis thaliana at the biochemical, transcriptomic, and proteomic levels. J Agr Food Chem. 2016;64:4497-504.

52. Song H, Lavoie M, Fan XJ, Tan HN, Liu GF, Xu PF, et al. Allelopathic interactions of linoleic acid and nitric oxide increase the competitive ability of Microcystis aeruginosa. ISME J. 2017;11:1865-76.

Ready to submit your research? Choose BMC and benefit from:

- fast, convenient online submission

- thorough peer review by experienced researchers in your field

- rapid publication on acceptance

- support for research data, including large and complex data types

- gold Open Access which fosters wider collaboration and increased citations

- maximum visibility for your research: over $100 \mathrm{M}$ website views per year

At $\mathrm{BMC}$, research is always in progress.

Learn more biomedcentral.com/submissions 